\title{
Spots on tooth enamel: what's new?
}

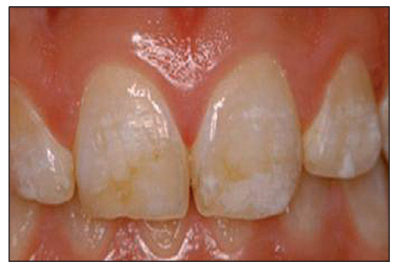

\author{
Muriel DE LA DURE-MOLLA, Chantal NAULIN-IFI, \\ Katia JEDEON, Ariane BERDAL, Sylvie BABAJKO
}

\begin{abstract}
Enamel is the most visible tissue of the tooth. It gives the tooth its whiteness, its brilliance and is the main focus of our attention from an esthetic point of view. Unfortunately, it is not uncommon to see alterations in this enamel, whether they are simple localized discolored spots, or more extensive loss of tooth substance. The etiologies, then, are multiple: toxicity during the mineralization of the bud, hyperthermia or a genetic abnormality. As professionals, making an etiological diagnosis and reassuring the parents is a challenge that we must address on a daily basis.
\end{abstract}

KEY WORDS

Dysplasia

Hypomineralization
Amelogenesis

Enamel
Even though the enamel is the hardest tissue of the tooth, paradoxically, it is also biologically the most brittle. In fact, defects in the enamel presenting most often as spots or as loss of tooth substances are much more frequent than abnormalities of the dentin or cement. From a terminological standpoint, any alteration in the enamel can be designated by the generic term "enamel dysplasia". This dysplasia is comprised of three distinct entities: hypoplasias, hypomineralizations and hypomaturations (Fig. 1).

- Hypoplasias are quantitative alterations caused by a defect of secretion of the enamel matrix. Clinically, this leads to a loss of localized tooth substance, or a decrease in thickness of enamel layer. The remaining enamel,
Address for correspondence:

Muriel de La Dure

Private practice

121 boulevard Jean Jaurès

92100 Boulogne Bilancourt

murielmolla@gmail.com

Article available at nttp://www.jdao-journal.org or nttp://dx.dc
Article received: 03-2013 Accepted for publication: 04-2013 


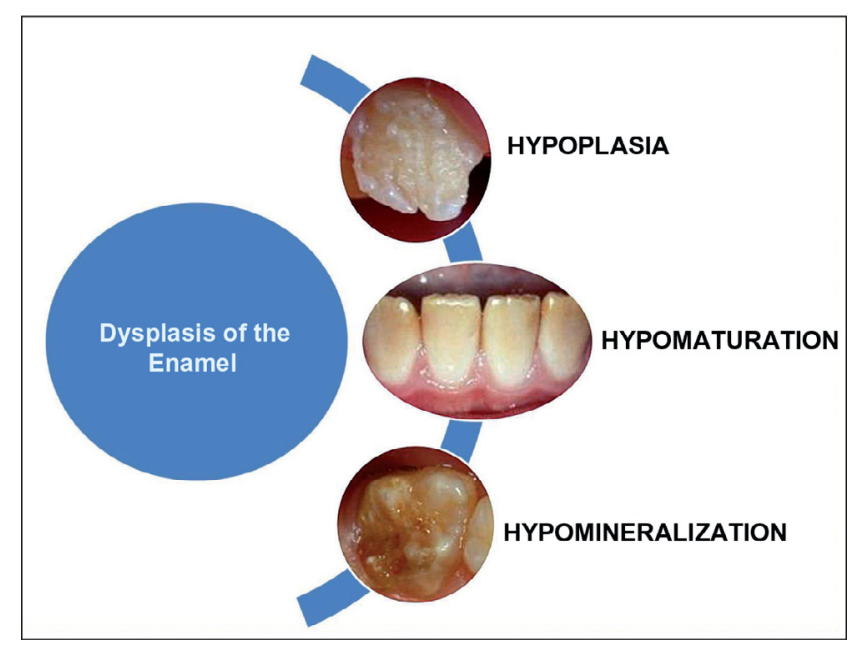

Figure 1

Summary diagram of the different clinical alterations in the enamel.

on the other hand, is very hard and its coloring is normal or slightly yellow.

- Hypomaturations and hypomineralizations are, conversely, qualitative defects where the very mineralization process itself is altered. The organic matrix is therefore excessive. Strictly speaking, with hypomaturation, the decrease in mineralization is less severe than in hypomineralization. Hypomaturation generally shows up as an alteration in the color of the enamel varying from chalky white to dark brown. On the other hand, there is no loss of enamel in the affected area.

- Hypomineralization is the most severe alteration of the enamel. The degree of mineralization is reduced which leads to brittle, soft when probed and rapidly worn enamel. It is a dark yellow or even orange color. The patient therefore complains about significant pain with changes in temperature and when chewing hard foods.

Each of these alterations corresponds to a histological defect occurring during specific stage of anamelogenesis. Hypoplasias arise during the secretion stage, hypomaturations during the maturation stage and hypomineralizations during the enamel extracellular matrix mineralization ${ }^{5}$.

The prevalence of defects in the enamel is greater in permanent teeth than in deciduous teeth, but extremely variable from one study to the next. In fact, the prevalence is from $24 \%$ to $49 \%$ (40.2\% in Spain) for temporary teeth and from $9 \%$ to $63 \%$ (52\% in Spain) for permanent teeth ${ }^{6}$. Of course, enamel defects arising in the permanent dentition concern parents the most, especially when they appear in the anterior sectors. Parents are concerned about various issues: the esthetics, the risk of seeing other teeth affected and the risk for brittleness of the affected tooth. But the first question that the parents ask is often "where did it come from"?

The etiologies are then extremely variable: traumatic, systemic and genetic. Clinical examination, medical history, questioning of the parents concerning the growth, life style and the home environment of the child provide information for positive and differential diagnosis. The first step of this diagnosis is to determine how many teeth are affected.

- When a single tooth is affected, the etiological factor is therefore local. It is necessary to discover if there was an earlier trauma that occurred to the primary tooth (especially, a traumatic intrusion). 
The root of the primary tooth will exert force on the bud of the permanent tooth during morphogenesis and therefore disturb the amelobastics layer. This type of trauma most frequently involves the incisors. The $2^{\text {nd }}$ possible local etiological factor is infection. In fact, if an infection presents on a primary molar and propagates to the succesional tooth during its morphogenesis, this will cause an abnormality in the permanent tooth that is then referred to as a "Turner's tooth". The premolars are teeth most affected by this disorder.

- When several teeth are affected, the etiology to consider therefore is systemic toxicity, that's mean prolonged exposure to a factor that alters amelogenesis.

\section{- Fluorosis:}

Fluoride was the first enamel toxin described. In fact, an overdose of fluoride, namely an intake of fluoride greater than $1.5 \mathrm{mg} / \mathrm{j}$, is responsible for dental fluorosis. This dosage is reached very quickly. In fact, by way of comparison, drinking water has a fluoride content on average varying From 0.3 to $0.5 \mathrm{mg} / \mathrm{l}$. The prophylactic dose is $0.05 \mathrm{mg} / \mathrm{kg} / \mathrm{j}$. The seriousness of enamel alteration varies, from "very mild" forms to "severe" forms according to Dean classification in 1942. The mildest form is characterized by limited small opaque areas affecting at least half of the dental surface and the severe form is characterized by brown or even black stains combined with areas of hypoplasia (Fig. 2). Excessive intake of fluoride generally occurs during the first years of a child's life. Fluorosis then affects all the teeth during

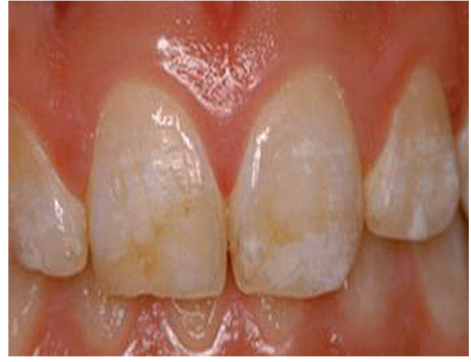

Figure 2

Intraoral view of a patient with mild to moderate dental fluorosis.

mineralization at that age, namely the permanent incisors, the $1^{\text {st }}$ permanent molars, some of the canines and premolars. The $2^{\text {nd }}$ molars are very rarely affected by fluorosis. Since this pathology is well known now, its prevalence decreased considerably ranging from $3.9 \%$ in 1991 to $2.73 \%$ in 1998 in France.

- MIH: Molar Incisor Hypomineralization:

In 2001, Weerheijm defined $\mathrm{MIH}$ as being a "hypomineralization of systemic origin frequently affecting 1-4 permanent first molars whether associated or not with affected permanent incisors"9. The damage can be mild, moderate or severe. In the mild forms, we see limited white or light yellow spots.

In moderate forms, these spots are more extensive, ranging from white to brown. And in the severe forms, these spots are associated with hypoplasias. The severity of the damage to the incisors generally indicates the severity of the molar involvement, whereas the converse is not true. Generally, the spots are well-defined on the incisors, especially impacting the buccal surface. At the level of the molars, on the 

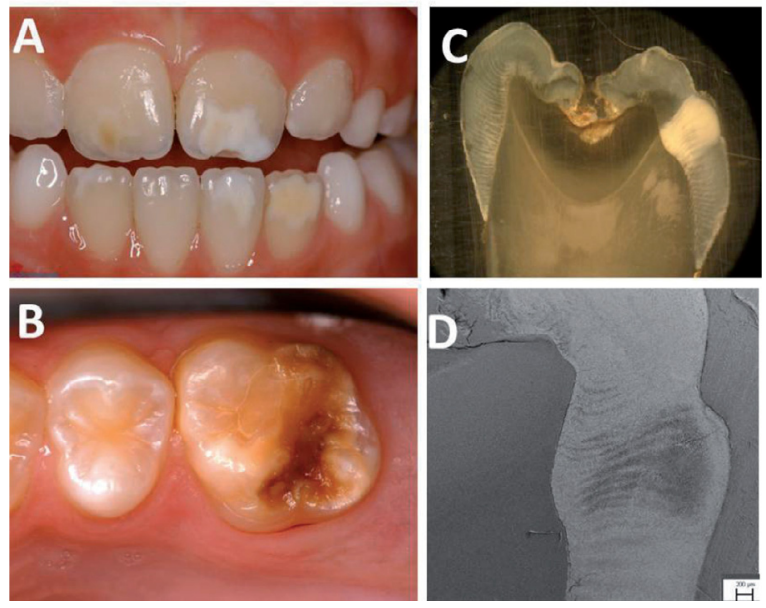

other hand, they are more diffuse and especially affecting the occlusal surface and the buccal surface. The damage is not symmetrical either in relation to the affected tooth nor in relation to the severity of the damage from one tooth to the other in the same patient. All the other permanent teeth are healthy, only the incisors and permanent first molars are affected. These hypomineralizations make the enamel extremely susceptible to wear and to caries (Fig. 3). The molars are the teeth most affected by this phenomenon of a carious lesion that then will develop very rapidly. It is not uncommon to see caries approaching the pulp on teeth that are still erupting. An histological analysis of these teeth confirms hypomineralization of the enamel in the affected area. Prisms are then separated from one another by the non-degraded organic matrix rendering the enamel permeable to microorganisms ${ }^{3}$.

The damage is always asymmetrical.
Figure 3

$a-b$ : Intraoral views showing un incisor and a permanent molar affected by $\mathrm{MIH}$. The incisors present white and yellows stains that are well defined. The molar presents a localized brown spot on the occlusal surface combined with a hypoplasia of the enamel on the bucco-distal surface of the cuspid.

c: Microscopic photograph of a cut of a tooth presenting a localized white spot on the palatal surface.

d: Scanning electron microscope photograph of the previous cut that visualizes a deformation in the thickness of the enamel in the area affected by MIH as well as a decrease in mineralization (dark gray areas).

Different criteria should be taken into account for the diagnosis:

- the presence of well-defined opacities either white, yellow or brown in color;

- post-eruptive losses of enamel due to abrasion of the hypomineralized enamel;

- atypical restorations;

- extractions of the first molars combined with damage to the incisors in a patient who presents a low risk for caries.

When the incisors are the only teeth affected, MIH can be ruled out.

The etiology of $\mathrm{MIH}$ is still unknown. Numerous epidemiological studies have been carried out, without really pinpointing any causal factor. The only certain finding is that one or more toxic factors occurred during the end of pregnancy or during the first years of life of the child. In fact, this period corresponds to the period of mineralization of these affected teeth. The different possible causes that have been men- 
tioned are: respiratory infections, complications at birth, dioxin, low birth weight, low oxygenation at birth, phosphocalcic disorders, early childhood sicknesses, antibiotics and prolonged maternal breastfeeding ${ }^{4}$. This pathology is becoming a real public health problem in recent years with an average prevalence of $18 \%$, and as high as $37.5 \%$ in certain studies.

Children affected by $\mathrm{MIH}$ are very anxious during consultation. The main reason is the pain that their molars cause after they erupt. Brushing is difficult, and the treatment for these teeth is 10 times more frequent than for any other tooth. The practitioner has to manage the child's anxiety and at the same time deal with technical difficulties: difficulties for the anesthesia, difficulties with the restoration (unclear limits between the healthy enamel and the pathological enamel). The treatment for the molars is the most challenging. The least affected teeth can be restored with composite materials. If the occlusal morphology is altered, pediatric crown can be placed. On the other hand, when the pulpal vitality of a tooth is affected for a child under the age of 9, long apexification procedures have to be performed since the first molars are still immature. If a panoramic xray shows that the permanent third molar is present, it's then better to extract the first molar and let the remaining two molars develop mesially.

- When all the teeth are affected, and this includes primary as well as permanent dentition, a genetic abnormality is the only possible etiology. A mutation of the gene involved in the process enamel formation could alter the synthesis, the mineralization or the architecture of the extracellular enamel matrix. This pathology is called hereditary amelogenesis imperfecta ${ }^{1}$. The enamel defect is as severe in the primary dentition as in permanent dentition and the alteration of the enamel is visible as soon as the tooth erupts into the oral cavity (Fig. 4). Although no epidemiological data are available in France, the prevalence of amelogenesis imperfecta in other parts of the world is very low ranging from $1 / 700$ to $1 /$ 14000 individuals. More than 10 genes could be the cause of amelogenesis imperfecta. Since genetic testing is not routinely performed in France, the diagnosis remains exclusively clinical. From a therapeutic point of view, there are multiple objectives: to prevent pain, to protect the permanent teeth (by maintaining the primary teeth) and functionally and esthetically rehabilitate the arches. In a surprising way, these patients do not present a high risk for caries. From an orthodontic standpoint however, we noticed a greater prevalence of skeletal open bite.

Orthodontic or orthopedic treatments are in no way contraindicated for enamel dysplasia or even for cases of amelogenesis imperfecta. Nonetheless, it is preferable to rehabilitate the teeth before beginning treatment. In fact, it has been shown that alterations in the enamel increase the risk for caries ${ }^{7}$. In the molar sectors, the pediatric crowns 

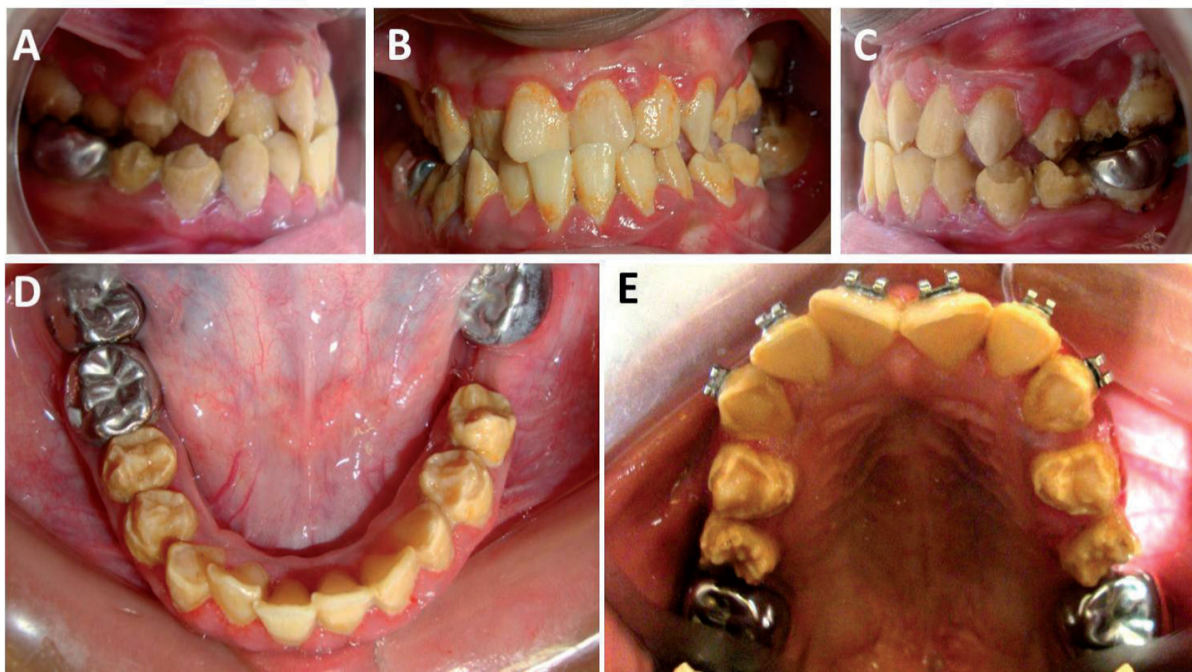

Figure 4

Intraoral photograph of a female patient in young adult dentition presenting the hypomineralized and hypoplastic type of hereditary amelogenesis imperfect.

make it possible to protect the teeth and not interfere with the placement of brackets. As for the bonding, it can be done with glass ionomer cement or composites following traditional protocol. For hypomature spots (brown), certain authors recommend to complete the acid etch with a sodium hypochorite rinse $(5 \%$ for 1 minute) in order to eliminate any organic material ${ }^{8}$.

Spots on the enamel are common but are discret most of the time. They are frequently the main reason for the concerns of the parents and it is important to be able to reassure them not only on the cause but also on the various treatments.

\section{REFERENCES}

1. Crawford PJ, Aldred M, Bloch-Zupan A. Amelogenesis imperfecta. Orphanet Journal of Rare Diseases 2007;2:17.

2. Gomes AC, Messias LP, Delbem AC, Cunha RF. Developmental disturbance of an unerupted permanent incisor due to trauma to its predecessor. Journal Canadian Dental Association 2010;76:a57.

3. Jedeon K, De la Dure-Molla M, Brookes SJ, Loiodice S, Marciano C, Kirkham J, Canivenc Lavier MC, Boudalia S, Bergés R, Harada H, Berdal A, Babajko S. Enamel defects reflect perinatal exposure to bisphenol A. Am J Pathol 2013 (sous presse).

4. Lygidakis NA, Dimou G, Marinou D. Molar-incisor-hypomineralisation (MIH). A retrospective clinical study in Greek children. II. Possible medical aetiological factors. Eur Arch Paediatr Dent 2008;9:207-217. 
5. Molla M, Naulin-Ifi, C, Berdal A. Enamel defects: frequence, aetiology and therapeutic aspect. Arch Pediatr 2010;17:758-759.

6. Robles MJ, Ruiz M, Bravo-Perez M, Gonzalez E, Penalver MA. Prevalence of enamel defects in primary and permanent teeth in a group of schoolchildren from Granada (Spain). Medicina Oral, Patologia Oral y Cirugia Bucal 2013;18:e187-193.

7. Targino AG, Rosenblatt A, Oliveira AF, Chaves AM, Santos VE. The relationship of enamel defects and caries: a cohort study. Oral Diseases 2011;17:420-426.

8. Venezie RD, Vadiakas G, Christensen JR, Wright JT. Enamel pretreatment with sodium hypochlorite to enhance bonding in hypocalcified amelogenesis imperfecta: case report and SEM analysis. Pediatric Dentistry 1994;16:433-436.

9. Weerheijm KL. Molar incisor hypomineralisation $(\mathrm{MIH})$. Eur $\mathrm{J}$ Paediatr Dent 2003;4:114-120. 